\title{
Optical MISO IM/DD Channels: Optimality of Spatial Repetition Codes among DC-offset STBCs
}

\author{
Yerzhan Sapenov, Anas Chaaban, Zouheir Rezki, and Mohamed-Slim Alouini
}

\begin{abstract}
In this paper, an optical wireless multipleinput single-output communication system employing intensitymodulation direct-detection is considered. Subject to a per transmit-aperture power constraint, the performance of direct current (DC) offset space-time block codes (STBC) is studied in terms of pairwise error probability (PEP). It is shown that among the class of DC-STBCs, the worst case PEP, i.e., the one corresponding to the minimum distance between two codewords, is minimized by repetition coding (RC) for any channel state. Therefore, it follows that among all DC-STBCs, RC is optimal in terms of worst case PEP under any turbulence statistics. This result agrees with previously published numerical results showing the superiority of RC in such systems. It also agrees with previously published analytical results on this topic under lognormal turbulence and further extends it to arbitrary turbulence statistics. Numerical results provided to verify this indicate that RC is not only optimal in terms of worst case PEP, but also in terms of average error probability.
\end{abstract}

\section{INTRODUCTION}

The continuous increase in the demand for higher data rates stresses current radio frequency (RF) wireless communication systems and requires new solutions for coping with this increase. One such a solution is optical wireless communications (OWC), in which light is used to convey information between communicating nodes [1]. OWC enjoys many attractive features including free broadband spectrum, enhanced security, and reduced electromagnetic interference, and therefore, it has witnessed increasing research attention from academic and industrial worlds recently. Furthermore, OWC operating in the visible-light spectrum, known as visible-light communications (VLC) [2], [3], allows simultaneous communication and illumination leading to increased power efficiency. These features make OWC a promising solution for future wireless communication systems, especially in its simple and practical intensity-modulated direct-detection (IM/DD) form.

Recent studies on IM/DD OWC focus on various performance criteria including achievable rates and capacity [4][7], outage probability [8], [9], and error rates [10]-[13], for instance. Diversity is one of the main performance criteria for reliable wireless communication [14], which can be achieved by exploiting channel variation in space, time, frequency, or

Y. Sapenov, A. Chaaban, and M.-S. Alouini are with the Computer, Electrical, and Mathematical Sciences and Engineering Division, King Abdullah University of Science and Technology (KAUST), Thuwal, Saudi Arabia. Email: \{yerzhan.sapenov,anas.chaaban,slim.alouini\} @kaust.edu.sa.

Z. Rezki is with the Department of Electrical and Computer Engineering, University of Idaho, Moscow, Idaho, USA. Email: zrezki@uidaho.edu.

This work was supported by the Qatar National Research Fund (a member of Qatar Foundation) under Grant NPRP9-077-2-036. The statements made herein are solely the responsibility of the authors. combinations thereof. Space and time can be exploited by proper design of space-time block codes (STBC) [15]. It is known that orthogonal STBC (OSTBC) are optimal in RF communications in terms of diversity gain. So the question which arises is: Does this also hold true in IM/DD OWC?

This question has been discussed in [11], where it was demonstrated numerically that spatial repetition coding $(\mathrm{RC})^{1}$ interestingly outperforms OSTBC in multiple input single output (MISO) IM/DD OWC systems with individual (i.e., per aperture) power constraint. RC is a special case of STBC where the temporal dimension is ignored and the transmit symbol is repeated spatially. In this paper, we aim to address this problem analytically, by studying the worst case pairwise error probability (PEP) of the class of direct current (DC) offset STBC in MISO IM/DD systems. We focus on the worst case PEP, corresponding to the minimum distance between any distinct codewords, since (i) it dictates the diversity order, and (ii) it can be used to obtain an upper bound and also an approximation of the actual error probability [16], [17].

Our contribution can be summarized as follows. For a MISO IM/DD system subject to an individual power constraint with BPSK alphabet, we prove that the worst case PEP of a DCSTBC is minimized by $\mathrm{RC}$ under given channel state for some block length. The proof is obtained by deriving an upper bound on the minimum distance of an arbitrary DC-STBC, and then showing that this upper bound is indeed achievable by using RC. The proof holds for both electrical and optical power constraints. We also provide a numerical evaluation which demonstrates that RC is not only optimal in terms of worst case PEP, but also in terms of error probability. Note that this result can be readily generalized to pulse amplitude modulation (PAM) rate-1 (1 PAM symbol/transmission) multiple input multiple output (MIMO) systems subject to an individual or a sum power constraint.

This proves the optimality of RC from worst case PEP point of view under a given channel state, which is of interest in indoors VLC where turbulence is absent. This also implies that $\mathrm{RC}$ is optimal from this point-of-view under any turbulence distribution. Note that the result is consistent with [12], [13] which prove the diversity-optimality of RC, among all space codes, for $M \times N$ MIMO system with log-normal distributed channels and a sum power constraints. The main difference with our work here is that we do not restrict our analysis to specific statistics, and that we also consider the temporal dimension in combination with the spatial one which leads to a more general result.

\footnotetext{
${ }^{1}$ Throughout the paper, we refer to spatial RC simply as RC.
} 


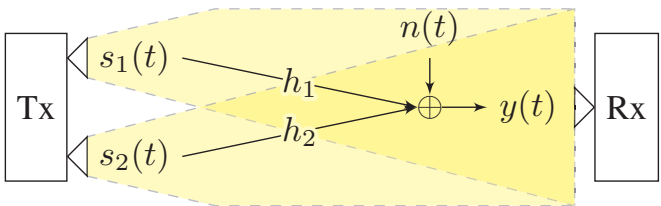

Fig. 1. A MISO OWC system with $N_{t}=2$ transmit apertures and one detector at time $t: s_{i}(t) \geq 0$ is the optical intensity of aperture $i, h_{i} \geq 0$ is the channel gain from aperture $i$ to the detector, and $n(t)$ is the Gaussian noise.

The rest of the paper is organized as follows. In Sec. II we introduce the system model and formulate the problem. The analysis of the problem and its solution are discussed in Sec. III under an electrical power constraint and given channel state. Then, in Sec. IV, we provide numerical simulations that verify the result, and extend the result to optical power constraints and turbulence scenarios. Finally, we conclude in Sec. V.

\section{System Model AND PROBlem Formulation}

\section{A. The IM/DD MISO OWC Channel}

Consider an IM/DD MISO OWC system consisting of $N_{t}$ transmit apertures and 1 receive aperture (see Fig. 1). The transmission model in time instant $t$ can be represented as

$$
y(t)=\mathbf{h}^{T} \mathbf{s}(t)+n(t),
$$

where $\mathbf{s}(t) \in \mathbb{R}_{+}^{N_{t} \times 1}$ is the vector of transmit signals, $\mathbf{h} \in$ $\mathbb{R}_{+}^{N_{t} \times 1}$ is the vector of the channel coefficients, and $n(t)$ is an additive white Gaussian noise with zero mean and variance $\sigma^{2}$, independent through time, and combines thermal noise and background radiation. The nonnegativity of $\mathbf{s}(t)$ and $\mathbf{h}$ follows since we consider IM/DD operation [18]. The channel state $\mathbf{h}$ is considered to be quasi-static, it remains constant for a block of $\mathrm{L}$ transmissions and changes independently in the next block. This is motivated by the fact the indoors VLC channels are generally not turbulent and outdoors free-space optical (FSO) communications vary very slowly in comparison to the symbol duration. It is assumed that the channel state information (CSI) is known at the receiver side.

The nonnegativity of $s_{i}(t)$, the $i^{\text {th }}$ symbol of $\mathbf{s}(t)$, can be guaranteed by using a DC offset. These symbols are constrained by individual power constraints. Common power constraints considered in this context are:

1) An electrical power constraint $\mathbb{E}\left[s_{i}^{2}(t)\right] \leq P \forall t$, or

2) An optical power constraint $\mathbb{E}\left[s_{i}(t)\right] \leq P \forall t$ [19].

Another possibility is to have a sum power constraint, which is not considered here due to space limitations. In what follows, we focus on an individual electrical power constraint. Then, we extend the result to the case with an optical power constraint. Since we are interested in the performance of DC-offset STBC in this paper, we define it next.

\section{B. DC-offset STBC}

Let $\mathbf{x} \in \mathbb{R}^{L \times 1}$ denote a vector of symbols that needs to be sent to the receiver in $L$ transmissions $t \in\{1, \ldots, L\}$, encoded in the transmit signals $\mathbf{s}(1), \ldots, \mathbf{s}(L)$. This is a rate-1 code, where one constellation symbol is sent per transmission

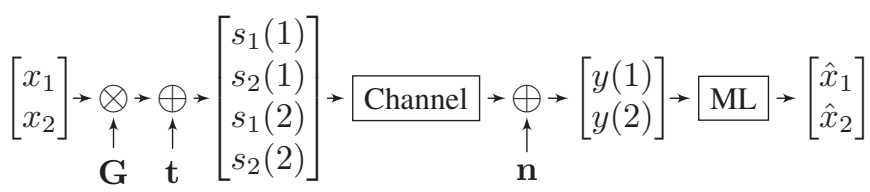

Fig. 2. Signal processing of $2 \times 1$ DC-STBC.

on average. For simplicity, we will restrict $\mathbf{x}$ to have BPSK symbols, i.e., $\mathbf{x} \in \mathcal{X} \triangleq\{-1,1\}^{L}$, although similar analysis can be applied for a more general PAM constellation. The DC-offset STBC is constructed as follows:

$$
\mathbf{s}=\mathbf{G x}+\mathbf{t},
$$

where $\mathbf{G}=\left[\mathbf{g}_{1}, \ldots, \mathbf{g}_{N_{t} L}\right]^{T} \in \mathbb{R}^{N_{t} L \times L}$ is the coding matrix, and $\mathbf{t}=\left[t_{1}, \ldots, t_{N_{t} L}\right]^{T} \in \mathbb{R}_{+}^{N_{t} L}$ is a DC-offset. Under this construction, the nonnegativity and individual electrical power constraints can be stated as follows ${ }^{2}$

$$
\begin{aligned}
\mathbf{g}_{i}^{T} \mathbf{x}+t_{i} & \geq 0 \\
\left\|\mathbf{g}_{i}\right\|_{2}^{2}+t_{i}^{2} & \leq P \text { for } i \in\left\{1, \ldots, N_{t} L\right\} .
\end{aligned}
$$

Because we consider BPSK, the minimum permissible DCoffset which guarantees the nonnegativity of $s$ is

$$
t_{i}=\left\|\mathbf{g}_{i}\right\|_{1}
$$

The received signal in the $L$ transmissions can be written as

$$
\mathbf{y}=[y(1), \ldots, y(L)]^{T}=\mathbf{H} \mathbf{s}+\mathbf{n},
$$

where $\mathbf{n}=[n(1), \ldots, n(L)]^{T}$, and

$$
\mathbf{H}=\left[\begin{array}{cccc}
\mathbf{h}^{T} & \mathbf{0}_{1 \times N_{t}} & \ldots & \mathbf{0}_{1 \times N_{t}} \\
\mathbf{0}_{1 \times N_{t}} & \mathbf{h}^{T} & \ldots & \mathbf{0}_{1 \times N_{t}} \\
\vdots & \vdots & \ddots & \vdots \\
\mathbf{0}_{1 \times N_{t}} & \mathbf{0}_{1 \times N_{t}} & \cdots & \mathbf{h}^{T}
\end{array}\right]
$$

is the extended-channel matrix with $\mathbf{0}_{1 \times N_{t}}$ being an allzero $N_{t}$-dimensional row vector. In what follows, we analyze the system for a given $\mathbf{h}$. The discussion on channels with turbulence is presented in Sec. III-C. The overall system can be rewritten as

$$
\mathbf{y}=\mathbf{H G} \mathbf{x}+\mathbf{H t}+\mathbf{n},
$$

as shown in Fig. 2 for $N_{t}=2$ and $L=2$. The goal is to design $\mathbf{G}$ so that the system performance is optimized in terms of the worst case PEP as discussed next.

\section{Problem Formulation}

Since channel states are known at the receiver and since the vector $\mathbf{t}$ is predefined, $\mathbf{H t}$ can be subtracted at the receiver side leading to the signal

$$
\dot{\mathbf{y}}=\mathbf{A x}+\mathbf{n},
$$

where $\mathbf{A}=\mathbf{H G}$. Due to the complexity of calculating the exact probability of error for a general $\mathbf{G}$, it is common to analyze the union bound [14]. It comprises the sum of the

\footnotetext{
${ }^{2}$ Throughout the paper, we use $\|\cdot\|_{2},\|\cdot\|_{1}$, and $\|\cdot\|_{F}$ to denote the $\ell_{2}$, $\ell_{1}$ and Frobenius norms, respectively.
} 
PEP, where the PEP between symbols $\mathrm{x}_{a}$ and $\mathrm{x}_{b}$ in $\mathcal{X}$ is defined as [20]

$$
P\left\{\mathbf{x}_{a} \rightarrow \mathbf{x}_{b} \mid \mathbf{H}\right\}=Q\left(\frac{\left\|\mathbf{A}\left(\mathbf{x}_{a}-\mathbf{x}_{b}\right)\right\|_{2}}{2 \sigma}\right),
$$

where $Q(\cdot)$ is the Q-function.

Our objective in this paper is to minimize the worst case PEP defined for a given $\mathbf{G}$ as

$$
\begin{aligned}
P_{e}(\mathbf{G}) & =\max _{\mathbf{x}_{a}, \mathbf{x}_{b} \in \mathcal{X}} Q\left(\frac{\left\|\mathbf{A}\left(\mathbf{x}_{a}-\mathbf{x}_{b}\right)\right\|_{2}}{2 \sigma}\right) \\
& =Q\left(\frac{\min _{\mathbf{x}_{a}, \mathbf{x}_{b} \in \mathcal{X}}\left\|\mathbf{A}\left(\mathbf{x}_{a}-\mathbf{x}_{b}\right)\right\|_{2}}{2 \sigma}\right),
\end{aligned}
$$

since $Q(x)$ is a decreasing function of $x$. One reason for choosing this objective is that the worst case PEP determines the diversity order of the system [17]. Another reason is that the exact symbol error rate (SER) can be approximated in terms of the worst case PEP using the union bound by $k_{\min } P_{e}(\mathbf{G})$ where $k_{\min }$ is the average number of neighboring code symbols (s) at the minimum distance [17]. This approximation is very accurate in the high SNR regime. The SER can also be upper bounded in terms of the worst case PEP by $(L-1) P_{e}(\mathbf{G})[16]$. Thus, minimizing the worst case PEP maximizes the diversity order, and minimizes the SER approximation and upper bound.

The minimum worst case pairwise error probability can be written as

$$
\begin{aligned}
P_{e, \min } & =\min _{\mathbf{G}} Q\left(\frac{\min _{\mathbf{x}_{a}, \mathbf{x}_{b} \in \mathcal{X}}\left\|\mathbf{A}\left(\mathbf{x}_{a}-\mathbf{x}_{b}\right)\right\|_{2}}{2 \sigma}\right) \\
& =Q\left(\frac{\max _{\mathbf{G}} \min _{\mathbf{x}_{a}, \mathbf{x}_{b} \in \mathcal{X}}\left\|\mathbf{A}\left(\mathbf{x}_{a}-\mathbf{x}_{b}\right)\right\|_{2}}{2 \sigma}\right),
\end{aligned}
$$

again since $Q(x)$ is a decreasing function of its argument $x$. Therefore, the optimization problem can be written as

$$
\begin{aligned}
\max _{\mathbf{G}} & \min _{\mathbf{x}_{a}, \mathbf{x}_{b} \in \mathcal{X}}\left\|\mathbf{A}\left(\mathbf{x}_{a}-\mathbf{x}_{b}\right)\right\|_{2}^{2} \\
\text { s.t. } & \left\|\mathbf{g}_{i}\right\|_{2}^{2}+\left\|\mathbf{g}_{i}\right\|_{1}^{2} \leq P \text { for } i \in\left\{1, \ldots, N_{t} L\right\},
\end{aligned}
$$

where the constraints follow from (3) and (4). We derive the optimal matrix $\mathbf{G}$ in the following section.

\section{Problem Analysis}

\section{A. Electrical Power Constraint}

The following theorem presents the solution of (12).

Theorem 1. The optimal BPSK DC-STBC (in terms of worst case PEP) for the MISO IM/DD channel with an individual electrical power constraint is $R C$ corresponding to

$$
\mathbf{G}^{[R C]}=\sqrt{\frac{P}{2}} \mathbf{I}_{L} \otimes \mathbf{1}_{N_{t} \times 1} \text {, and } \mathbf{t}^{[R C]}=\sqrt{\frac{P}{2}} \mathbf{1}_{N_{t} L \times 1},
$$

where $\mathbf{I}_{L}$ is the $L \times L$ identity matrix, $\mathbf{1}_{N_{t} \times 1}$ is an $N_{t} \times 1$ vector of ones, and $\otimes$ is the Kronecker product. The corresponding minimum worst case PEP is given by

$$
P_{e, \min }=Q\left(\sqrt{\frac{P}{2 \sigma^{2}}\|\mathbf{h}\|_{1}^{2}}\right) .
$$

Proof: We can rewrite the problem in the following form

$$
\Theta^{[e]} \triangleq \max _{\mathbf{G} \in \mathcal{G}^{[e]}} \min _{\mathbf{x}_{a}, \mathbf{x}_{b} \in \mathcal{X}}\left\|\mathbf{A}\left(\mathbf{x}_{a}-\mathbf{x}_{b}\right)\right\|_{2}^{2},
$$

where

$\mathcal{G}^{[e]}=\left\{\mathbf{G} \in \mathbb{R}^{N_{t} L \times L} \mid\left\|\mathbf{g}_{i}\right\|_{2}^{2}+\left\|\mathbf{g}_{i}\right\|_{1}^{2} \leq P, i=1, \ldots, N_{t} L\right\}$.

We first upper bound $\Theta^{[e]}$, and then show that the resulting upper bound is indeed achievable leading to the optimal G. We start by considering all possible combinations of $\mathbf{x}_{a}, \mathbf{x}_{b} \in \mathcal{X}$ to analyze the objective of (15). Define

$$
\Psi_{a b}=\left\|\mathbf{A}\left(\mathbf{x}_{a}-\mathbf{x}_{b}\right)\right\|_{2}^{2} .
$$

Then, we have

$\Psi_{a b} \in S_{\Delta} \triangleq\left\{\left\|\sum_{i=1}^{L} \delta_{i} \mathbf{a}_{i}\right\|_{2}^{2} \mid \delta_{i} \in\{0,2,-2\}, i=1, \ldots, L\right\}$,

where $\mathbf{a}_{i}$ is the $i$ th column of matrix $\mathbf{A}$. Now, consider the following subset of $S_{\Delta}$

$$
S_{\Delta}^{\prime}=\left\{4\left\|\mathbf{a}_{i}\right\|_{2}^{2}, i=1, \ldots, L\right\} \subseteq S_{\Delta} .
$$

Note that

$$
\min _{\mathbf{x}_{a}, \mathbf{x}_{b} \in \mathcal{X}}\left\|\mathbf{A}\left(\mathbf{x}_{a}-\mathbf{x}_{b}\right)\right\|_{2}^{2}=\min _{\Psi_{a b} \in S_{\Delta}} \Psi_{a b} \leq \min _{\Psi_{a b} \in S_{\Delta}^{\prime}} \Psi_{a b} .
$$

But the minimum of a set is not larger than its average, i.e.,

$$
\min _{\Psi_{a b} \in S_{\Delta}^{\prime}} \Psi_{a b} \leq \frac{1}{L} \sum_{i=1}^{L} 4\left\|\mathbf{a}_{i}\right\|_{2}^{2}=\frac{4}{L}\|\mathbf{A}\|_{F}^{2},
$$

Thus, we have

$$
\Theta^{[e]} \leq \hat{\Theta}^{[e]} \triangleq \max _{\mathbf{G} \in \mathcal{G}^{[e]}} \frac{4}{L}\|\mathbf{A}\|_{F}^{2} .
$$

Let us write $\mathbf{G}$ as $\mathbf{G}=\left[\mathbf{F}(1)^{T}, \ldots, \mathbf{F}(L)^{T}\right]^{T}$, where $\mathbf{F}(i) \in$ $\mathbb{R}^{N_{t} \times L}, i \in\{1, \ldots, L\}$. Thus, $\mathbf{A}$ can also be written as

$$
\mathbf{A}=\left[\mathbf{A}(1)^{T}, \ldots, \mathbf{A}(L)^{T}\right]^{T},
$$

where $\mathbf{A}(i)=\mathbf{h}^{T} \mathbf{F}(i) \in \mathbb{R}^{1 \times L}, i \in\{1, \ldots, L\}$. This allows us to split (20) into $L$ independent sub problems, each of which depends on $N_{t}$ rows of $\mathbf{G}$ corresponding to $\mathbf{F}(i)$ for $i \in\{1, \ldots, L\}$. In particular,

$$
\hat{\Theta}^{[e]}=\max _{\mathbf{G} \in \mathcal{G}^{[e]}} \frac{4}{L} \sum_{k=1}^{L}\|\mathbf{A}(k)\|_{F}^{2}=\sum_{k=1}^{L} \max _{\mathbf{F}(k) \in \mathcal{F}^{[e]}} \frac{4}{L}\|\mathbf{A}(k)\|_{F}^{2},
$$

where $\mathcal{F}^{[e]}$ is the following set of matrices

$$
\mathcal{F}^{[e]}=\left\{\mathbf{F} \in \mathbb{R}^{N_{t} \times L} \mid\left\|\mathbf{f}_{i}\right\|_{2}^{2}+\left\|\mathbf{f}_{i}\right\|_{1}^{2} \leq P, i=1, \ldots, N_{t}\right\} .
$$

and $\mathbf{f}_{i}$ is the $i^{\text {th }}$ column of $\mathbf{F}$. Since these subproblems are similar, we focus on one of them given by

$$
\hat{\Theta}_{k}^{[e]} \triangleq \max _{\mathbf{F}(k) \in \mathcal{F}^{[e]}} \frac{4}{L}\|\mathbf{A}(k)\|_{F}^{2}=\max _{\mathbf{F}(k) \in \mathcal{F}[e]} \frac{4}{L} \sum_{i=1}^{L}\left(\mathbf{h}^{T} \mathbf{f}_{i}(k)\right)^{2} .
$$

Since the channel gains are nonnegative reals, then, a necessary condition on the optimal solution $\mathbf{F}(k)$ is that the 
components of $\mathbf{f}_{i}(k)$ have the same sign. ${ }^{3}$ By symmetry, we can restrict the components of $\mathbf{f}_{i}(k)$ to positive values. Furthermore, we can upper bound the problem by relaxing the constraints in (21) using the fact that $\left\|\mathbf{f}_{i}(k)\right\|_{2}^{2} \leq\left\|\mathbf{f}_{i}(k)\right\|_{1}^{2}$. Thus, we obtain $\hat{\Theta}_{k}^{[e]} \leq \tilde{\Theta}_{k}^{[e]}$ where $\tilde{\Theta}_{k}^{[e]}$ is the relaxed problem given by

$$
\tilde{\Theta}_{k}^{[e]} \triangleq \max _{\mathbf{F}(k) \in \mathcal{F}_{R}^{[e]}} \frac{4}{L}\|\mathbf{A}(k)\|_{F}^{2},
$$

where

$$
\mathcal{F}_{R}^{[e]}=\left\{\mathbf{F} \in \mathbb{R}^{N_{t} \times L} \mid\left\|\mathbf{f}_{i}\right\|_{2}^{2} \leq \frac{P}{2}, i=1, \ldots, N_{t}\right\} .
$$

The maximum of this relaxed problem is achieved when only one column of matrix $\mathbf{F}(k)$ has nonzero values. To show this, consider a feasible $\mathbf{F}(k)$ with two nonzero columns $\mathbf{f}_{1}(k)$ and $\mathbf{f}_{2}(k)$, and consider another feasible $\mathbf{F}^{\prime}(k)$ with only one nonzero column $\mathbf{f}_{1}^{\prime}(k)$ satisfying

$$
f_{1 i}^{\prime}(k)=\sqrt{f_{1 i}^{2}(k)+f_{2 i}^{2}(k)}, \forall i \in\left\{1, \ldots, N_{t}\right\},
$$

where $f_{1 i}^{\prime}(k), f_{1 i}(k)$, and $f_{2 i}(k)$ are the $i^{\text {th }}$ components of $\mathbf{f}_{1}^{\prime}(k), \mathbf{f}_{1}(k)$, and $\mathbf{f}_{2}(k)$, respectively, so that the rows of $\mathbf{F}(k)$ and $\mathbf{F}^{\prime}(k)$ have the same $\ell_{2}$-norm. One can easily verify that

$$
\left(\mathbf{h}^{T} \mathbf{f}_{1}(k)\right)^{2}+\left(\mathbf{h}^{T} \mathbf{f}_{2}(k)\right)^{2} \leq\left(\mathbf{h}^{T} \mathbf{f}_{1}^{\prime}(k)\right)^{2} .
$$

This approach can be used to show that an $\mathbf{F}^{\prime}(k)$ with one nonzero column is better than any $\mathbf{F}(k)$ with more than one nonzero column. Thus, the optimal solution of (22) must have only one nonzero column in $\mathbf{F}(k)$. Substituting in (22) yields

$$
\begin{aligned}
\tilde{\Theta}_{k}^{[e]}= & \max _{\mathbf{f}_{1}(k)} \frac{4}{L}\left(\mathbf{h}^{T} \mathbf{f}_{1}(k)\right)^{2} \\
& \text { s.t. } f_{1 i}^{2}(k) \leq \frac{P}{2}, \forall i \in\left\{1, \ldots, N_{t}\right\} .
\end{aligned}
$$

Since the objective is increasing with elements of $\mathbf{f}_{1}(k)$, then it is maximized when the constraints are met with equality. This implies that (25) is maximized by $\mathbf{f}_{1}(k)=\sqrt{\frac{P}{2}} \mathbf{1}_{N_{t} \times 1}$ leading to $\tilde{\Theta}_{k}^{[e]}=\frac{2 P}{L}\|\mathbf{h}\|_{1}^{2}$. Consequently, we have upper bounded (15) as follows

$$
\Theta^{[e]} \leq \hat{\Theta}^{[e]}=\sum_{k=1}^{L} \hat{\Theta}_{k}^{[e]} \leq \sum_{k=1}^{L} \tilde{\Theta}_{k}^{[e]}=2 P\|\mathbf{h}\|_{1}^{2} .
$$

This upper bound is achievable by setting $\mathbf{G}$ to (13) as in Theorem 1, which concludes the proof.

Next, we state a similar result under an individual optical power constraint, and then discuss systems with turbulence.

\section{B. Optical Power Constraint}

If the system is constrained with an optical power constraint instead of an electrical power constraint, then the problem can be formulated as follows

$$
\begin{aligned}
\max _{\mathbf{G}} & \min _{\mathbf{x}_{a}, \mathbf{x}_{b} \in \mathcal{X}}\left\|\mathbf{A}\left(\mathbf{x}_{a}-\mathbf{x}_{b}\right)\right\|_{2}^{2} \\
\text { s.t. } & \left\|\mathbf{g}_{i}\right\|_{1} \leq P \text { for } i \in\left\{1, \ldots, N_{t} L\right\} .
\end{aligned}
$$

\footnotetext{
${ }^{3}$ This is the main difference between this problem and one which arises in the RF context where the channels are complex-valued, which leads to a different solution.
}

By following a similar approach as in Sec. III-A, we can obtain the following theorem.

Theorem 2. The optimal BPSK DC-STBC (in terms of worst case PEP) for the MISO IM/DD channel with an individual optical power constraint is RC corresponding to

$$
\mathbf{G}^{[R C]}=P \mathbf{I}_{L} \otimes \mathbf{1}_{N_{t} \times 1}, \quad \text { and } \quad \mathbf{t}^{[R C]}=P \mathbf{1}_{N_{t} L \times 1} .
$$

The corresponding minimum worst case PEP is given by

$$
P_{e, \min }=Q\left(\sqrt{\frac{P^{2}}{\sigma^{2}}\|\mathbf{h}\|_{1}^{2}}\right) .
$$

Proof: The proof is similar to Sec. III-A and is omitted due to space limitations.

\section{Application for Static and Turbulent Channels}

So far we discussed the result for a single block transmission. However, in practice, transmission takes place over multiple blocks. Consider a transmission duration of $n=B L$ channel uses, where $B$ is the number of blocks of length $L$. In this case, the worst case PEP (defined for a BPSK vector of length $L$ transmitted over one block) needs to be averaged over all $B$ blocks. Thus, the criterion of interest is the minimum average worst case PEP, $\bar{P}_{e, \min }$.

For the static case, this averaging is redundant, i.e., the minimum average worst case PEP under an electrical power constraint is given by $\bar{P}_{e, \min }=Q\left(\sqrt{\frac{P}{2 \sigma^{2}}}\|\mathbf{h}\|_{1}\right)$, similar to the result in Theorem 1. Under an optical power constraint, the minimum average worst case PEP is $\bar{P}_{e, \min }=$ $Q\left(\sqrt{\frac{P}{2 \sigma^{2}}}\|\mathbf{h}\|_{1}\right)$, similar to the result in Theorem 2 .

On the other hand, under turbulence, $\mathbf{H}$ defined in (6) is a random variable which changes from block to block. In this case, the minimum average worst case PEP satisfies

$$
\bar{P}_{e, \min }=\min _{\mathbf{G}} \mathbb{E}_{\mathbf{H}}\left[P_{e}(\mathbf{G}) \mid \mathbf{H}\right] \geq \mathbb{E}_{\mathbf{H}}\left[\min _{\mathbf{G}} P_{e}(\mathbf{G}) \mid \mathbf{H}\right] .
$$

But, under an electrical power constraint, this lower bound is achievable by setting $\mathbf{G}$ and $\mathbf{t}$ as in Theorem 1 , which do not depend on $\mathbf{H}$. Thus, $\mathrm{RC}$ is optimal as it minimizes the average worst case PEP above. A similar statement holds under an optical power constraint.

This result is applicable for any turbulence distribution, and is in agreement with [11]. Compared to [12], our result is more general since it (i) holds for any turbulence distribution and not restricted to log-normal statistics and (ii) considers both spatial and temporal coding and shows the redundancy of the temporal dimension. From another perspective, our result is less general than [12] since we consider MISO with a individual power constraints (similar to [11]) while [12] studies MIMO with a sum power constraint.

\section{Discussion AND NumERICAL EVAluATION}

Let us consider the special case $N_{t}=2$ with individual electrical power constraints. The exact SER $p(\mathbf{G})$ of the system employing BPSK can be bounded in terms of the worst case PEP as follows [16]

$$
\frac{1}{2} P_{e}(\mathbf{G}) \leq p(\mathbf{G}) \leq 3 P_{e}(\mathbf{G})
$$




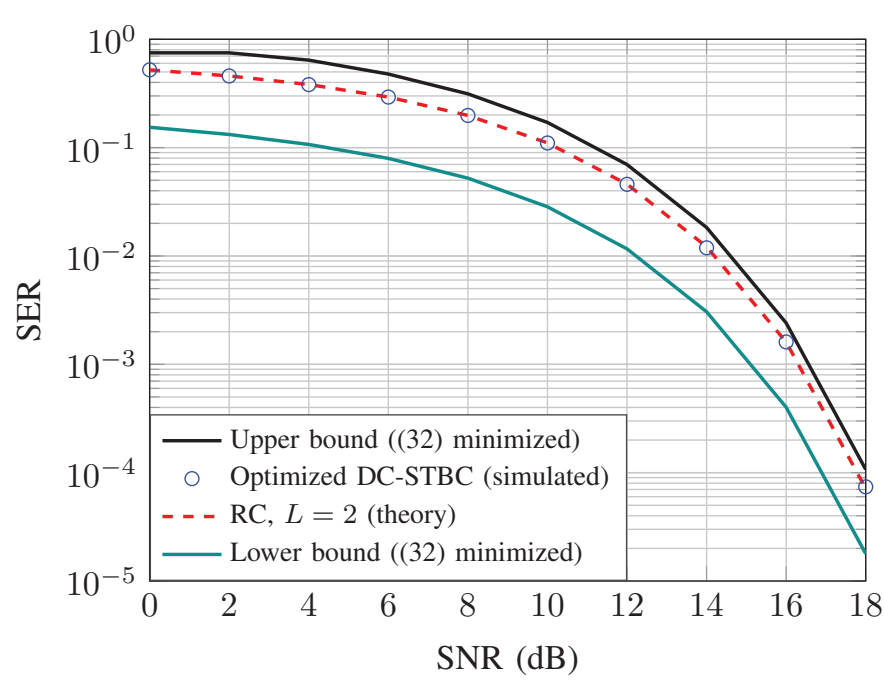

Fig. 3. SER performance comparison of a $2 \times 1$ MISO OWC system with channels $h_{1}(1)=h_{1}(2)=0.2$ and $h_{2}(1)=h_{2}(2)=0.3$.

By minimizing the worst case PEP, we also minimize the bounds. For the optimal solution which is RC, the SER can be expressed in terms of the worst case PEP as follows

$$
p\left(\mathbf{G}^{[R C]}\right)=1-\left(1-P_{e}\left(\mathbf{G}^{[R C]}\right)\right)^{2} \approx 2 P_{e}\left(\mathbf{G}^{[R C]}\right),
$$

where $\mathbf{G}^{[R C]}$ is given in (13), and the approximation is accurate for $P_{e}\left(\mathbf{G}^{[R C]}\right) \ll 1$.

Numerical comparisons are provided in Fig. 3. In this figure, we show the minimized SER upper and lower bounds given in (32), the SER of RC $p\left(\mathbf{G}^{[R C]}\right)$ given in (33), and the SER of DC-STBC $p(\mathbf{G})$ minimized numerically with respect to G. The upper bound provides a fairly tight approximation of the SER at high SNR (electrical, $P / \sigma^{2}$ ). The minimized SER of DC-STBC $\min _{\mathbf{G}} p(\mathbf{G})$ coincides with the SER of RC $p\left(\mathbf{G}^{[R C]}\right)$. As predicted, the numerically calculated optimal $\mathbf{G}$ which minimizes $p(\mathbf{G})$ for DC-STBC corresponds to RC and $k_{\min }=2$. This demonstrates the optimality of RC not only with respect to the worst case PEP, but also with respect to the SER for $N_{t}=2$.

\section{CONClusion}

In this paper, we studied a MISO IM/DD OWC system employing DC-offset STBC. We considered the worst case PEP as a main criterion for performance evaluation. We analyzed the problem of minimizing this criterion under individual electrical power constraints. We proved the optimality of spatial repetition coding for the $N_{t} \times 1$ MISO case. This result means that the temporal dimension is redundant in such systems as far as the worst case PEP is concerned, and that it is enough to code spatially. It is particularly interesting since repetition coding is simpler in practice. We extended the result to the case with optical power constraints. We also discussed applications of this result for static channels (e.g. indoors VLC) and turbulent channels (outdoors FSO). The result will be generalized to sum electrical/optical power constraints and also to MIMO systems in future work.

\section{REFERENCES}

[1] M. A. Khalighi and M. Uysal, "Survey on free space optical communications: A communication theory perspective," IEEE Commun. Surveys Tuts., vol. 16, no. 4, pp. 2231-2258, 4th quarter 2014.

[2] H. Haas, L. Yin, Y. Wang, and C. Chen, "What is LiFi?" J. Lightw. Technol., vol. 34, no. 6, pp. 1533-1544, Mar. 2016.

[3] H. Elgala, R. Mesleh, and H. Haas, "Indoor optical wireless communication: Potential and state-of-the-art," IEEE Comm. Magazine, vol. 49, no. 9, pp. 56-62, Sep. 2011.

[4] R. Jiang, Z. Wang, Q. Wang, and L. Dai, "A tight upper bound on channel capacity for visible light communications," IEEE Commun. Letters, vol. 20, no. 1, pp. 97-100, Jan. 2016.

[5] A. Lapidoth, S. M. Moser, and M. Wigger, "On the capacity of freespace optical intensity channels," IEEE Trans. Inf. Theory, vol. 55, no. 10, pp. 4449-4461, Oct. 2009.

[6] A. Chaaban, Z. Rezki, and M.-S. Alouini, "On the capacity of the intensity-modulation direct-detection optical broadcast channel," IEEE Trans. Wireless Commun., vol. 15, no. 5, pp. 3114-3130, May 2016.

[7] A. Chaaban, J.-M. Morvan, and M.-S. Alouini, "Free-space optical communications: Capacity bounds, approximations, and a new spherepacking perspective," IEEE Trans. Commun., vol. 64, no. 3, pp. 11761191, Mar. 2016.

[8] N. Letzepis and A. G. i Fabregas, "Outage probability of the freespace optical channel with doubly stochastic scintillation," IEEE Tran. Commun., vol. 57, no. 10, pp. 2899-2902, Oct. 2009.

[9] A. Chaaban, Z. Rezki, and M.-S. Alouini, "Fundamental limits of parallel optical wireless channels: Capacity results and outage formulation," IEEE Trans. Commun., vol. 65, no. 1, pp. 296-311, Jan. 2017.

[10] T. Tsiftsis, H. G. Sandalidis, G. K. Karagiannidis, and M. Uysal, "Optical wireless links with spatial diversity over strong atmospheric turbulence channels," IEEE Trans. Wireless Commun., vol. 8, no. 2, pp. 951-957, Feb. 2009.

[11] M. Safari and M. Uysal, "Do we really need OSTBCs for free-space optical communication with direct detection?" IEEE Trans. Wireless Commun., vol. 7, no. 11, pp. 4445-4448, Nov. 2008.

[12] Y. Y. Zhang, H. Y. Yu, J. K. Zhang, Y. J. Zhu, J. L. Wang, and X. S. $\mathrm{Ji}$, "On the optimality of spatial repetition coding for MIMO optical wireless communications," IEEE Commun. Letters, vol. 20, no. 5, pp. 846-849, May 2016.

[13] Y. Y. Zhang, H. Y. Yu, J. K. Zhang, Y. J. Zhu, J. L. Wang, and T. Wang, "Full large-scale diversity space codes for MIMO optical wireless communications," in 2015 IEEE Int. Symp. Inf. Theory, June 2015, pp. 1671-1675.

[14] D. Tse and P. Viswanath, Fundamentals of Wireless Communications. Cambridge University Press, 2005.

[15] V. Tarokh, N. Seshadri, and A. R. Calderbank, "Space-time codes for high data rate wireless communication: Performance criterion and code construction," IEEE Trans. Inf. Theory, vol. 44, no. 2, pp. 744-765, Mar. 1998.

[16] M. Salehi and J. Proakis, Digital Communications. McGraw-Hill Education, 2007.

[17] J. R. Barry, D. G. Messerschmitt, and E. A. Lee, Digital Communication: Third Edition. Norwell, MA, USA: Kluwer Academic Publishers, 2003.

[18] S. Hranilovic and F. R. Kschischang, "Optical intensity-modulated direct detection channels: signal space and lattice codes," IEEE Trans. Inf. Theory, vol. 49, no. 6, pp. 1385-1399, June 2003.

[19] J. Armstrong and B. J. C. Schmidt, "Comparison of asymmetrically clipped optical OFDM and DC-Biased optical OFDM in AWGN," IEEE Commun. Letters, vol. 12, no. 5, pp. 343-345, May 2008.

[20] G. L. Stüber, Principles of Mobile Communication, 3rd ed. Springer Publishing Company, Incorporated, 2011. 